\title{
L'ÉVOLUTION DES TRADUCTIONS DE L'ART DE LA TRADUCTION ET SA RÉCEPTION À L'ÉTRANGER ${ }^{1}$
}

\author{
Elizaveta GETTA \\ Université Charles de Prague ${ }^{2}$
}

\begin{abstract}
En): The article deals with the genesis of Jiři Levý's monograph The Art of Translation, one of his most important works and one of the fundamental works of Czech translatology. It discusses the original Czech version, its different later editions, translated versions that have appeared over time, and the foreign reception of this work.
\end{abstract}

Keywords (En): Jiří Levý ; The Art of Translation ; evolution ; versions

Mots-clés (Fr) : Jiří Levý; L'Art de la traduction; évolution; versions

DOI : $10.32725 /$ eer.2021.018

L'Art de la traduction (Umění překladu, 1963)33 est un ouvrage de Jiř́ Levý, le plus grand théoricien de la traduction tchèque, dont le contenu tout comme l'évolution sont remarquables. Divisé en deux parties, le livre porte essentiellement sur la traduction littéraire. La première partie est consacrée à la théorie générale de la traduction tandis que la seconde se focalise sur la traduction de la poésie. Levý commence par mettre en lumière la différence entre les approches linguistiques de la traduction et celles qui découlent de la critique littéraire. Il décrit ensuite trois phases principales du travail de traducteur, chacune de leurs étapes et de leurs difficultés. Il continue en abordant la question des problèmes esthétiques de la traduction, en particulier la dialectique entre la norme de la reproduction et de l'esthétique, mais aussi les divergences qui séparent la culture de départ et la culture d'arrivée. Entre autres, Levý dresse une typologie détaillée des erreurs de traduction les plus fréquentes tout en en décrivant les conséquences. La première partie du livre se clôt sur quelques pages consacrées à la traduction des titres, puis sur un chapitre entier portant sur la traduction théâtrale. Dans la seconde partie du livre, l'auteur

\footnotetext{
${ }^{1}$ L'article a d'abord été rédigé en tchèque puis traduit en français par Zuzana Št'astná. Sa version originale a été publiée en 2021 dans la revue Svět literatury. GETTA Jelizaveta (2021), «Geneze a zahraniční recepce Umění překladu Jiř́ho Levého ", in: Svět literatury 18/1: 9-25. [En ligne]. https://svetliteratury.ff.cuni.cz/wp-content/uploads/sites/14/2021/06/Jelizaveta_Getta_9-25.pdf [consulté le 17 novembre, 2021].

${ }^{2}$ Dédicace : La rédaction du présent article a été rendue possible grâce à la subvention de GA UK n. 68420 intitulée «Překladatelská evoluce Umění překladu: Komparativní analýza českého originálu a jeho německého překladu, doplněná o komentáŕe překladatele ke spolupráci s autorem, Jiř́ím Levým " [L'Évolution des traductions de l'Art de la traduction: Analyse comparative de l'original tchèque et de sa traduction allemande accompagnée par des commentaires du traducteur concernant la collaboration avec l'auteur de l'œuvre, Jiř́ Levý] et effectuée à la Faculté des Lettres de l'Université Charles. Affiliation : Jelizaveta Getta, Faculté des Lettres de l’Université Charles.

${ }^{3}$ L'ouvrage n'a pas encore été traduit en français.
} 
détaille plusieurs approches relatives à la traduction de différents types de vers et systèmes métriques.

D'autre part, il introduit de nombreuses notions théoriques: à savoir la traductivité, les traductions illusionniste et anti-illusionniste, la norme de traduction et d'autres qu'il illustre ensuite d'exemples concrets. L'œuvre de Jiř́ Levý est non seulement digne d'intérêt en vertu de son esprit novateur, mais également pour son style d'écriture captivant. Le lecteur ne se sent pas gêné par la terminologie ; parfois, il peut même oublier qu'il est en train de lire une théorie de la traduction.

L'original tchèque de L'Art de la traduction paraît en 1963, éveillant aussitôt l'attention à l'étranger. C'est en 1969 que la première traduction, en allemand, voit le jour. À cette époque-là, les travaux sur sa version russe sont déjà en cours. La rédaction des deux nécessite d'importantes modifications sur les plans linguistique et pragmatique, car de nombreux éléments présents dans l'original sont étroitement liés au milieu littéraire tchèque. Afin d'en faciliter la traduction, Levý modifie la version originale de son ouvrage d'une façon considérable : il remplace notamment les exemples tchèques illustrant des phénomènes de traduction par des analogies allemandes et russes. En outre, la publication est actualisée et complétée par de nouveaux acquis. Ainsi, la traduction allemande rend pour la première fois les idées de Levý accessibles à l'étranger. Concurremment avec la version originale, elle sert plus tard en tant que fondement pour la deuxième édition tchèque de L'Art de la traduction, préparée en 1983 sous la plume de Karel Hausenblas. Bien que près de soixante ans se soient écoulés depuis sa première parution, les notions et réflexions théoriques présentes dans cet ouvrage ne perdent rien de leur actualité, au contraire. Au cours des dix dernières années, la publication a été traduite (en totalité ou en partie) en d'autres langues; de nos jours, elle est en cours de relecture et de redécouverte. En 2011, Patrick Corness et Zuzana Jettmarová ont traduit L'Art de la traduction en langue anglaise. Pour ce projet, le traducteur et la rédactrice se sont appuyés sur la deuxième édition tchèque préparée par Karel Hausenblas ainsi que sur la traduction allemande. Ils ont consulté également certains passages de l'édition russe.

Le présent article a pour objectif d'étudier la genèse de L'Art de la traduction et de décrire les différentes étapes de sa création dans les contextes national tchèque et international, en se focalisant en premier lieu sur la francophonie. Nous allons nous pencher également sur la genèse de la traduction allemande en la confrontant avec les éditions tchèques. Les données de la recherche sont collectées suivant les méthodes de la recherche empirique qualitative suivantes : la recherche en archives, l'analyse traductologique et l'entretien semi-directif.

\section{L'original de L'Art de la traduction}

Les travaux sur la première édition de L'Art de la traduction (1963) datent déjà des années cinquante. Cette monographie peut être considérée comme le point culminant de l'activité scientifique interdisciplinaire à laquelle Levý s'adonne depuis longtemps. ${ }^{4}$

\footnotetext{
${ }^{4}$ L'Art de la traduction allie les acquis de la linguistique structurale à l'esthétique. $-76-$
} 
Les fondements élaborés de L'Art de la traduction apparaissent pour la première fois en $1953^{5}$ dans l'article intitulé «O některých zákonitostech překladatelské věrnosti » [Quelques principes de la fidélité en traduction], publié dans la revue tchèque Slovo a slovesnost. "Překladatelský proceses - jeho objektivní podmínky a psychologie » [Le processus de la traduction - ses conditions objectives et sa psychologie $]^{6}$ est un autre article significatif publié en 1955 dans la même revue. Il sera plus tard inséré dans L'Art de la traduction et devient la base du polycopié Kapitoly z teorie a metodiky překladu [Chapitres de la théorie et des méthodes de traduction], créé en collaboration avec Bohumil Ilek et publié en 1956.

En 1958, Levý publie un autre polycopié intitulé Úvod do teorie prekladu [Introduction à la théorie de la traduction]. Il s'agit cette fois-ci d'une monographie qui reproduit la structure de l'article «Umělecké otázky překladu » [Les questions esthétiques de la traduction] paru en 1957 dans la revue Česká literatura et que Levý commente ainsi : «J'assemble ici certains de mes écrits sur la théorie de la traduction littéraire pour les rendre accessibles en recueil car ils forment un ensemble cohérent. $\gg{ }^{7}$ Cette publication comporte quatre chapitres et évoque déjà, d'une façon très nette, la première partie de son ouvrage suivant, L'Art de la traduction. Vladimír Rossels, qui a traduit l'ouvrage crucial de Levý en russe, désigne ce polycopié comme l'une des deux étapes fondamentales menant à la naissance de L'Art de la traduction.

Après sa première publication en 1963, l'ouvrage a été retravaillé et complété par un précis de littérature traitant de la problématique donnée, des recherches détaillées sur les genres de la littérature traduite et leurs particularités, une partie dédiée à la versologie et des exemples d'analyses concrètes visant des problèmes de la traduction. Les chapitres déjà existants ont été considérablement élargis, le nombre de pages se multipliant ainsi par deux. C'est également à ce moment-là que l'auteur a minutieusement hiérarchisé la structure de son écrit. ${ }^{8}$

Rossels considère l'édition de 1963 plutôt comme un pas vers une version internationale de L'Art (voir le chapitre « Les versions allemande et russe de L'Art de la traduction » pour plus de détails). ${ }^{9}$

Il est nécessaire de mentionner ici deux travaux de recherche fondamentaux pour le parcours scientifique de Levý. Il s'agit de sa thèse de doctorat «Vývoj překladatelských metod V české literatuře » ([L’Évolution des méthodes de traduction dans la littérature tchèque], 1957) et de sa thèse de maître de conférences « Základní otázky teorie překladu » ([Les questions fondamentales de la théorie de la traduction], 1958), dans le cadre desquelles l'auteur a effectué un travail de recherche indispensable à la rédaction de L'Art de la traduction. L'ouvrage reflète également des résultats du travail comparatif auquel Levý se consacre depuis longtemps. Dans L'Art de la traduction, il présente des dizaines d'études analysant

\footnotetext{
${ }^{5}$ Levý 1953, p. 63-80.

${ }^{6}$ Levý 1963, p. 65-87.

${ }^{7}$ LEVÝ, 1958, p. 3.

${ }^{8}$ Rossels in LeVÝ, 1974, p. 10-11, trad. J. G.

${ }^{9}$ Rossels in LeVÝ, 1974, p. 13.
} 
le style d'expression unique de nombreux auteurs. À titre d'exemple, citons les noms de Karel Čapek, Walt Whitman ou William Shakespeare. ${ }^{10}$

\section{Les versions allemande et russe de L'Art de la traduction}

La première édition de L'Art de la traduction suscite l'intérêt immédiat de la communauté scientifique étrangère, à commencer par la République démocratique allemande. La maison d'édition de Francfort Athenäum confie la traduction à Walter Schamschula en raison de ses études en slavistique, de son bilinguisme et de son origine tchéco-allemande. Schamschula affirme avoir entamé la traduction de L'Art en 1965. La traduction en langue russe se déroule en parallèle avec la traduction allemande, ${ }^{11}$ tâche attribuée à Vladimir Rossels ; elle est publiée en 1974 par la maison d'édition moscovite Progress.

Schamschula déclare que la nature de L'Art de la traduction a été problématique pour les traducteurs car l'original se concentre sur l'analyse de la littérature et de la traduction tchèques. D'après Rossels, c'est la raison pour laquelle les traducteurs désirant travailler sur ce livre ont demandé à Levý de modifier le caractère des exemples cités et de rendre le texte plus accessible aux lecteurs et lectrices ciblés. ${ }^{12}$

Dans la préface de l'édition allemande, Schamschula s'exprime également sur le processus de la traduction en collaboration avec l'auteur. Selon Schamschula, Levý a coopéré avec lui de façon enthousiaste. Ils se sont notamment consultés sur des questions de terminologie : en effet, au vu du caractère novateur de L'Art de la traduction, l'absence des termes appropriés dans la langue allemande a obligé à en inventer. Schamschula indique également que Levý a en même temps travaillé sur les adaptations allemande et russe. ${ }^{13}$

En outre, Rossels évoque la possibilité d'une version internationale de L'Art de la traduction:

Ainsi, Levý s'est mis à envisager une troisième variante de l'ouvrage; cette fois-ci internationale - d'abord, il n'a voulu qu'échanger les exemples cités, mais ensuite il s'est rendu compte du fait que chaque modification de ce type nécessiterait de vastes aménagements et par la suite des conclusions spécifiées. ${ }^{14}$

\footnotetext{
${ }^{10}$ Par exemple : LEVÝ Jiří (1956), « Čapkův Loupežník a Shakespeare », Jazyk a literatura 3, Sborník Vysoké školy pedagogické v Olomouci, Praha, SPN ou : LeVÝ Jiří (1955), «Walt Whitman v českých překladech », Host do domu 11, no 2, p. 513-515.

${ }^{11}$ Schamschula en juge par la publication de Levý Západní literární véda a estetika [La science littéraire et l'esthétique à l'Ouest] qui contient une dédicace datée de 1966, l'année de leur rencontre à Brno (information reçue par courrier électronique le 7 juillet 2020). Dans la préface de la version russe, Rossels indique avoir débattu de la traduction avec Levý en 1965. Certains chapitres de L'Art de la traduction sont traduits l'année suivante et publiés dans le bulletin Tetradi perevodčika (Rossels 1974, p. 13).

12 RoSSELS in LeVÝ, 1974, p. 13.

${ }^{13}$ SCHAMSChUla in LeVÝ, 1969, p. 11.

${ }^{14}$ Rossels in LevÝ, 1974, p. 13, trad. J. G. 
Nous pouvons donc nous demander s'il en existait deux versions différentes (des manuscrits préliminaires complets sont parfois mentionnés), respectivement pour l'édition allemande et russe, ou bien si Levý a voulu retravailler son livre pour donner naissance à une version internationale. Schamschula affirme ne se souvenir d'aucun manuscrit préliminaire complet de L'Art de la traduction qui aurait dû servir de modèle pour l'édition allemande. Il s'agissait selon lui de pages à part dont il a considérablement fallu modifier le contenu pour les rendre accessibles au public allemand. En rédigeant la traduction, Schamschula a travaillé à partir de l'original publié en Tchécoslovaquie en $1963 .{ }^{15}$

Les sources qui confirment la coopération entre Schamschula et Levý, tout en n'excluant pas l'intention de ce dernier de créer une version internationale du livre, se trouvent dans les archives de l'Université Masaryk de Brno. Il s'agit de collages composés à partir de coupures de la première édition tchèque. Levý avait rayé des passages qu'il jugeait non pertinents pour le public international et y avait intercalé des propositions par lesquelles il aurait été possible de remplacer les passages laissés de côté. Il s'est parfois contenté de retravailler les pages de la première édition (1963). À titre d'exemple, nous pourrions citer la page n. $62^{16}$ portant en version originale sur la relation ambiguë qu'a une traduction par rapport aux littératures des cultures originale et cible. Levý propose de supprimer un passage décrivant la situation littéraire durant le Renouveau national tchèque. Il remplace ces informations par un paragraphe dédié aux interprétations de l'œuvre de François Villon dans la période d'entre-deux-guerres. Cette modification est ensuite incorporée dans la version allemande de L'Art de la traduction. En outre, Levý enlève un paragraphe dans lequel il explique que la traduction peut non seulement renforcer, voire remplacer la littérature originale, mais aussi la concurrencer. Il soutient son hypothèse en citant la littérature appartenant à l'époque du Renouveau national. Pourtant, dans la traduction allemande cette modification n'est pas tout à fait respectée - seuls les exemples liés au milieu tchèque sont omis. ${ }^{17}$

La plupart des documents préparatoires se trouvent dans le dossier «Zbytky z německé verze (při ruské úpravě) » [Ce qui reste de la version allemande (conçue d'après l'édition russe)]. Les pages retravaillées sont enrichies d'exemples provenant de la littérature allemande, tandis que les extraits issus de la littérature russe ne sont pas effacés. Prenons l'exemple de la page n. 137, où l'auteur souligne le caractère ambigu du langage utilisé dans les dialogues théâtrales : il le documente en citant un extrait de "Tableau de famille», comédie écrite par Alexandre Ostrovski, qui apparaît dans les deux versions. Dans l'édition allemande, l'interprétation tchèque de l'extrait est supprimée et remplacée par une traduction allemande,$^{18}$ tandis qu'en édition russe elle est conservée et simplement complétée par des explications en russe. ${ }^{19}$ En constatant que Levý a décidé d'incorporer les exemples provenant de la littérature russe à la version allemande, Schamschula

\footnotetext{
${ }^{15}$ Entretien avec Walter Schamschula, Skype : Prague-Huntsville, le 17 juin 2020.

${ }^{16}$ La pagination dans les documents d'archives correspond à l'édition de 1963.

${ }^{17}$ LevÝ, 1969, p. 75-76. Archives de l’Université Masaryk de Brno, Fonds personnel de Jiř́i Levý, B 34 - Notes sur la théorie de la traduction, Carton 2.

${ }^{18}$ LEVÝ, 1969, p. 149.

${ }^{19}$ LeVÝ, 1974, p. 204-205.
} 
confirme que les deux versions ont pu être créées en parallèle. Nous pouvons donc supposer que Levý avait pour objectif de créer une compilation destinée aux lecteurs internationaux..$^{20}$ De plus, l'aspect graphique de l'édition allemande correspond à celui de l'édition russe. À titre d'exemple, nous allons encore une fois nous servir d'une section du chapitre « La traduction des pièces de théâtre », « Перевод пьес » en russe. ${ }^{21}$ Dans les deux cas, l'auteur a utilisé le même schéma pour illustrer la façon dont les traducteurs ont travaillé avec la syntaxe difficile d'Hamlet. ${ }^{22}$

\section{Les particularités de la traduction allemande de L'Art de la traduction}

Le présent chapitre vise à déterminer les spécificités liées à la traduction de $L$ 'Art de la traduction vers l'allemand. Elles peuvent être divisées en deux groupes : les spécificités du contenu et du contexte. Les premières consistent en une forte dépendance de l'original à l'égard de la culture tchèque. Les problèmes les plus brûlants se situent sur les plans lexical et pragmatique.Les spécificités du contexte sont quant à elles liées à la situation politique d'alors et à l'interruption de la coopération entre le traducteur et l'auteur due à la mort précoce de ce dernier.

Le niveau lexical est représenté avant tout par des termes transposés en langue allemande pour la première fois, ce que reflète l'inconsistance dans la traduction de certains d'entre eux. Penchons-nous sur la notion de la « traductivité ». ${ }^{23}$ C'est l'un des néologismes de Levý, repris et développé plus tard par Anton Popovič. ${ }^{24}$ Levý propose deux définitions de la «traductivité », à savoir : 1) le fait que l'œuvre est traduite, ou 2) une valeur de la traduction, qui peut être positive ou négative selon la qualité de la traduction concernée. Schamschula utilise le terme «das Übersetztsein », ${ }^{25}$ signifiant « être traduit ». Dans un autre contexte il emploie l'adjectif nominalisé « das Übersetzerische » désignant « ce qui se rapporte à la traduction $» .{ }^{26}$

Suivant les documents d'archives, Levý avait à sa disposition le manuscrit de la traduction allemande. Il avait effectué des corrections que Schamschula affirme ne jamais avoir reçues. ${ }^{27}$ Une correction du manuscrit allemand contenant des modifications par rapport au lexique, aux signes diacritiques et à l'aspect graphique faites par l'auteur lui-même, se trouve dans les archives personnelles de Jiř́ Levý. Les interventions de grande envergure, comme les additions ou les omissions, sont sporadiques. Cependant, force est de constater que certains passages dans la traduction finale diffèrent de ce que l'on trouve dans le manuscrit ainsi que dans les corrections proposées. ${ }^{28}$ Étudions la phrase suivante : «L'ouvrage ne se réalise

\footnotetext{
${ }^{20}$ Correspondance par courriel avec Walter Schamschula, le 7 juillet 2020.

${ }^{21}$ LEVÝ, 1974, p. 178.

${ }^{22}$ En traduction allemande l'extrait se trouve à la page 128, en traduction russe à la page 180 .

${ }^{23}$ LEVÝ, 1963, p. 60.

${ }^{24}$ PopOVIČ, 1983, p. 177.

${ }^{25}$ LEVÝ, 1969, p. 74.

${ }^{26}$ LEVÝ, 1969, p. 76.

${ }^{27}$ Correspondance par courriel avec Walter Schamschula, le 7 juillet 2020.

${ }^{28} \mathrm{Il}$ est donc possible qu'il ne s'agisse pas de la dernière correction avant l'impression du livre ou bien qu'elle ne soit pas parvenue aux mains de l'éditeur ou du traducteur.
} 
dans la société et n'a un effet esthétique que lorsqu'il est lu. $»^{29} \mathrm{La}$ version manuscrite de la traduction allemande contenait cette phrase : «Der Text eines Werks wird in der Gesellschaft der Leser realisiert und wirkt als Kunstwerk erst dann, wenn er gelesen wird.» Levý l'a corrigée en proposant de remplacer "Gesellschaft der Leser» (traduction littérale: "société des lecteurs ») par «Leserpublikum» (traduction littérale : « lectorat»). ${ }^{30}$ Cependant, c'est la formulation «Kulturmilieu des Lesers » (traduction littérale : « le milieu culturel du lecteur $»)$ qui se trouve dans la traduction finale. ${ }^{31}$

$\mathrm{Du}$ point de vue pragmatique, ce sont avant tout les exemples concrets accompagnant les thèses théoriques de Levý dans L'Art de la traduction qu'il a fallu adapter. Levý les a modifiés selon leur pertinence vis-à-vis du public allemand. Il a notamment essayé de trouver les analogies les plus exactes des phénomènes de traduction dans la littérature traduite en allemand et en tchèque.

Dans le chapitre en version originale « L'Interprétation de l'original », Levý démontre la subjectivité du traducteur à l'aide d'un extrait de "La Mégère apprivoisée » traduit en tchèque par Erik Adolf Saudek. "Saudek remplace les alentours de la ville de Stratford par une région tchèque où il est né. Il invente des noms de villages. $\gg{ }^{32}$ Quant au milieu germanophone, l'auteur découvre une tendance similaire chez Heinrich Zschokke. Un extrait dactylographié de sa traduction des Précieuses ridicules se trouve parmi les documents d'archives à l'Université Masaryk. Levý y ajoute une remarque disant que ce dernier pourrait remplacer l'exemple tchèque de E. A. Saudek. Il étudie et énumère les manières dont se manifeste la subjectivité du traducteur dans le travail de Zschokke. Alors que seuls les goûts littéraires de Zschokke transparaissent dans l'édition allemande, influençant son travail de traducteur, ${ }^{33}$ les corrections de Levý, quant à elles, font directement référence aux exemples de germanisation faites par Zschokke et qui correspondent à l'approche de Saudek mentionnée dans l'original de L'Art de la traduction. On peut dès lors se demander si l'éditeur ou le traducteur a tenu compte de cette information avant la parution officielle de la version allemande. ${ }^{34}$

Ce sont non seulement les spécificités du contenu, mais aussi celles du contexte qui sont cruciales dans le processus de la traduction. Selon une inscription à la fin du manuscrit, la traduction allemande aurait déjà dû être publiée en 1967. À en juger d'après les corrections, le manuscrit allemand est alors presque complet sans pour autant être prêt à la publication..$^{35}$ Dans la préface de Schamschula écrite autour de l'année 1968, il indique que la dernière chose que Levý a eu le temps de faire était une préface de l'édition allemande, ou plutôt le chapitre initial «La situation

${ }^{29}$ LEVÝ, 196, p. 20, surligné. J. G.

${ }^{30}$ Archives de l'Université Masaryk de Brno, Fonds personnel de Jiří Levý, B 34 - Notes sur la théorie de la traduction, Carton 1.

${ }^{31}$ LEVÝ, 1969, p. 37.

${ }^{32}$ LEVÝ, 1963, p. 32.

${ }^{33}$ LEVÝ, 1969, p. 48-49.

${ }^{34}$ Archives de l'Université Masaryk de Brno, Fonds personnel de Jiř́i Levý, B 34 - Notes sur la théorie de la traduction, Carton 4

${ }^{35}$ Archives de l'Université Masaryk de Brno, Fonds personnel de Jiří Levý, B 34 - Notes sur la théorie de la traduction, Carton 4. 
actuelle dans le domaine des théories de la traduction » (en allemand : «Der Stand der theoretischen Beschäftigung mit den Fragen des Übersetzens »). ${ }^{36}$ Levý a actualisé ce chapitre pour l'édition allemande afin qu'il reflète le développement international dans ce domaine du point de vue linguistique et littéraire. ${ }^{37}$

La mort de Levý au début de l'année 1967 influence les publications des deux traductions, aussi bien russe qu'allemande. En effet, lorsque Rossels travaillait sur la traduction russe de L'Art, Levý lui envoyait des chapitres individuels adaptés au lectorat russe. Les derniers d'entre eux sont arrivés à Moscou après le décès de l'auteur. ${ }^{38}$ Schamschula mentionne qu'après le départ subit de l'auteur, le manuscrit est ponctué de quelques passages manquant de clarté alors qu'une consultation aurait été nécessaire. Il finalise donc la traduction sans l'aide de Levý, mais en conformité avec ses idées et son style d'expression. De plus, Rossels actualise la bibliographie internationale relative aux théorie et pratique de la traduction. Il se sert de l'original tchèque et des documents que Levý lui avait offerts lors de leur collaboration.

Il est à noter que la création de l'édition allemande, basée sur la coopération entre l'auteur et le traducteur, est très probablement influencée par la situation politique d'alors. Schamschula présume que sa correspondance avec Levý a été surveillée et limitée par la censure. Nous pouvons donc supposer que certains documents envoyés par l'auteur ne sont jamais parvenus jusqu'à Francfort. ${ }^{39}$

\section{La deuxième édition tchèque de L'Art de la traduction}

La deuxième édition tchèque de L'Art de la traduction est publiée en 1983 par Karel Hausenblas. Linguiste multidisciplinaire, il s'intéresse également à la théorie et à la pratique de la traduction. Preuve en est son article «Styl a překlad» [Style et traduction],${ }^{40}$ une critique de L'Art de la traduction (voir ci-dessous), ainsi que ses archives personnelles qui contiennent notamment les documents relatifs à la théorie de la traduction et issus de l'Université Karl Marx à Leipzig. ${ }^{41}$

Hausenblas se prononce sur L'Art en 1965 dans une critique intitulée «Překládání a teorie literatury (Nad knihou J. Levého Uměni préekladu)» $[\mathrm{La}$ traduction et la théorie de la littérature (Sur la publication L'Art de la traduction de J. Levý)], écrit qu'il considère lui-même plutôt comme un essai. Entre autres, il reproche à Levý l'utilisation de l'expression «traduction réaliste ». "La notion d'une traduction réaliste n'y est guère définie et j'avoue ne pas être sûr de sa vraie signification ». Il aborde ici les confusions liées à l'absence de définition d'une traduction « irréaliste » ainsi que le manque de datation liée à ladite notion. Il est donc impossible, dit Hausenblas, de déterminer s'il s'agit du réalisme au sens large,

\footnotetext{
${ }^{36}$ Entretien avec Walter Schamschula, Skype : Prague-Huntsville, le 17 juin 2020.

${ }^{37}$ SCHAMSCHULA in LeVÝ, 1969, p. 11.

${ }^{38}$ RosSELS in LEVÝ, 1974, p. 16.

${ }^{39}$ Entretien avec Walter Schamschula, Skype : Prague-Huntsville, le 17 juin 2020.

${ }^{40}$ Document source : Hausenblas Karel (1967/68), «Styl a překlad », Dialog 11, nº 1, p. 13-36.

${ }^{41}$ Archives de l'Université Charles, Fonds personnel de Karel Hausenblas. À titre d'exemple citons le texte de Gert Jäger : Zur Problematik von Gegenstand und Objekt, ou de Otto Kade : Kommunikationswissenschaftliche Probleme der Translation.
} 
l'adjectif «réaliste» signifiant «n'importe quel art sain et progressiste de toute l'histoire », ou bien du réalisme au sens plus étroit, à savoir le courant «qui a dominé notre littérature pendant les dernières décennies du XIX ${ }^{\mathrm{e}}$ siècle ». ${ }^{42}$

Levý réagit à ce reproche dans la version retravaillée de $L^{\prime}$ 'Art destinée au public allemand. Il insère une explication dans le chapitre initial «Die literaturwissenschaftlichen Methoden » (dans la deuxième édition tchèque : « Les Méthodes de la science littéraire ») :

La notion du « réalisme » peut être interprétée soit au sens de l'histoire littéraire (en tant que méthode élaborée aux XVIII ${ }^{\mathrm{e}}$ et XIX ${ }^{\mathrm{e}}$ siècle par des auteurs réalistes), soit au sens philosophique (en tant qu'attitude épistémologique correspondant au matérialisme dialectique). ${ }^{43}$

Certains auteurs soviétiques utilisent ce terme pour remplacer le mot « adéquat », ajoute Levý tout en admettant que dans L'Art de la traduction la notion de « réalisme » est privée de sa signification concrète. ${ }^{44}$ La première édition du livre contient un chapitre à part nommé «Le Réalisme dans la traduction », ${ }^{45}$ rebaptisé pour la version allemande. Dans la deuxième édition tchèque il s'intitule «Les Problèmes esthétiques de la traduction $\gg{ }^{46}$ Le contenu reste le même, ce qui prouve que dans la première édition, Levý utilise l'adjectif « réaliste » pour désigner tout ce qui est « adéquat» ou bien " véridique ».

Hausenblas continue en critiquant l'analogie qu'établit Levý entre l'art dramatique et la traduction, car selon Hausenblas le but d'une pièce de théâtre est d'être mise en scène, tandis qu'une œuvre littéraire n'est pas a priori destinée à être traduite. Il se montre réticent à propos de la distinction entre « le texte de l'œuvre et l'œuvre au sens plus étroit» et juge inopportun de considérer le « mot» comme la plus petite unité linguistique. Toutefois, aucune édition future de L'Art de la traduction ne prendra ces reproches en considération. Étant donné le rapport ambivalent qu'a Hausenblas envers l'ouvrage de Levý, la question suivante surgit : quelle est son approche lors du travail sur la seconde édition de L'Art de la traduction? Dans sa préface, Hausenblas la commente ainsi :

Lors de la rédaction nous avons décidé de nous servir, dans la mesure du possible, de l'édition allemande. Cela n'a pas été facile, car cette édition vise des lecteurs différents, des lecteurs d'une autre langue, d'une autre littérature et d'un autre milieu. En même temps, cela aurait été une mauvaise décision de juste compléter la première version du texte par ce qui avait été ensuite ajouté pour l'édition allemande, et ce qui aurait pu intéresser le lectorat tchèque. En effet, l'auteur avait effectué d'autres changements importants $[\ldots] .{ }^{47}$

Hausenblas ajoute : « Nous ne sommes pas obligés d'être d'accord avec toutes les thèses que Jiř́ Levý propose dans son livre. En effet, dans les critiques de L'Art

\footnotetext{
42 Hausenblas, 1965, p. 161-162.

${ }^{43}$ LEVÝ, 1983, p. 34-35.

${ }^{44}$ LEVÝ, 1983, p. 34-35.

${ }^{45}$ LEVÝ, 1963, p. 49.

${ }^{46}$ LeVÝ, 1983, p. 82.

${ }^{47}$ Hausenblas in LevÝ, 1983, p. 10.
} 
de la traduction, pour la plupart positives, les évaluateurs formulent déjà quelques reproches [...]. » Il admet ensuite qu'il a été impossible d'éviter toute subjectivité lors du choix des éléments à garder et à éliminer. Néanmoins, l'éditeur a tâché de réduire ses propres formulations au minimum. Ses interventions sont très peu nombreuses, leur objectif principal étant de rendre le texte plus cohérent possible.

L'analyse comparative met en lumière l'effort réalisé par Hausenblas afin de conserver toutes les idées de la première édition de L'Art (1963) absentes dans la traduction allemande (1969) et de les incorporer à la deuxième édition tchèque (1983). Cela concerne par exemple cette citation de Otokar Fischer : «La traduction doit être suffisamment libre pour être fidèle $[\ldots] »{ }^{48}$ issue du chapitre « La traduction en tant qu'une sorte d'art » dans l'original de 1963. Levý la met en relation avec les traductions du tchèque vers le slovaque et vice versa. ${ }^{49}$

En même temps, nous remarquons la volonté de Hausenblas de ne pas priver la nouvelle édition des informations présentes dans la version allemande. Comme il est indiqué dans la préface, il est au courant de l'existence d'une version du manuscrit modifiée par Levý pour être adaptée au public allemand, mais sans l'avoir à sa disposition..$^{50}$ Concernant le chapitre «Le processus de la traduction » (1963), expliquant trois étapes du travail de traducteur, le texte en version allemande est enrichi d'une représentation de ce processus en tant que double chaîne de communication, ${ }^{51}$ qui apparaît ensuite dans la seconde édition thèque (1983). ${ }^{52}$

En guise de récompense pour son travail éditorial, Karel Hausenblas est nominé pour le prix du Český literární fond (Fondation tchèque pour la littérature) par la maison d'édition Panorama. Le directeur de la maison d'édition le justifie en soulignant l'importance de son travail et les nombreux problèmes qu'il a dû surmonter. Le plus problématique, selon lui, est de combiner les deux textes (la version originale tchèque et la traduction allemande) pour les faire fusionner de manière naturelle. ${ }^{53}$

La seconde édition (1983) sert comme base pour deux éditions suivantes de L'Art de la traduction. En 1998 l'ouvrage est publié par la maison d'édition Ivo Železný. D'après une note éditoriale il s'agit d'une réimpression sans interventions majeures. ${ }^{54}$

La plus récente, la quatrième édition est publiée en 2012 par la maison d'édition Apostrof et préparée par Miroslav Pošta. Lors de son travail d'éditeur, Miroslav Pošta consulte non seulement les deuxième et troisième éditions de L'Art, mais également sa traduction en langue anglaise parue en 2011. Étant donné le contexte différent, il supprime certaines mentions de la philosophie marxiste et corrige des erreurs dans les extraits en langue étrangère. ${ }^{55}$

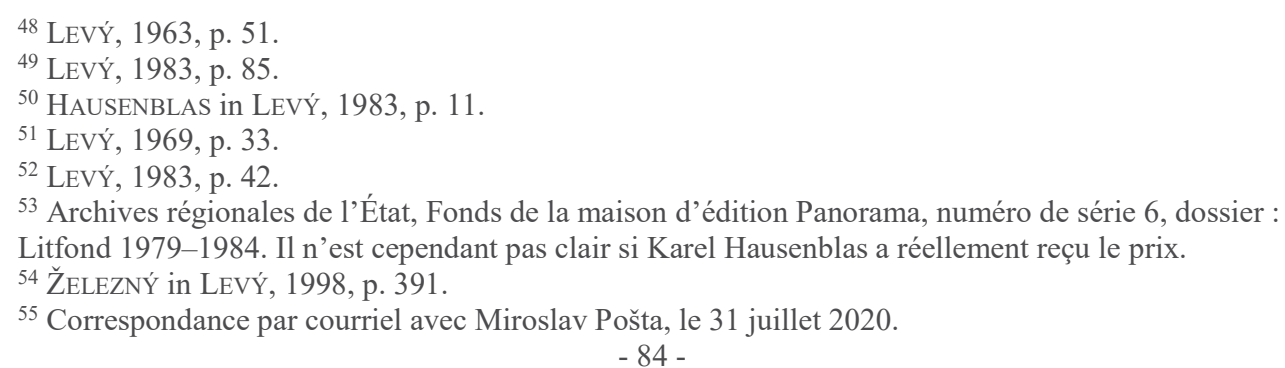




\section{La réception de L'Art de la traduction à l'étranger}

La première édition de L'Art de la traduction en langue étrangère, Die literarische Übersetzung: Theorie einer Kunstgattung (1969) devient à la fois une source essentielle pour la deuxième édition tchèque et rend les idées de Levý accessibles au public étranger. En 1991, José Lambert constate que la version allemande de cet ouvrage a changé la conception occidentale de la traduction et de la littérature traduite. ${ }^{56}$

Les idées de Levý influencent notamment la théorie du polysystème et l'école de manipulation. Dans les années soixante-dix, le travail de Levý inspire un groupe de chercheurs affiliés à James Holmes. Ce dernier connaît bien (dans le cadre de ses possibilités linguistiques) tout ce qui se passe en traductologie à l'international. Ses publications prouvent que l'œuvre de Levý lui est familière. Les normes de reproduction et de l'esthétique introduites par le théoricien tchèque ressemblent beaucoup à deux règles fondamentales pour la traduction de la poésie proposées par Holmes. D'après lui, une telle traduction doit être à la fois suffisamment fidèle à l'original pour être considérée comme une traduction, et esthétique pour être considérée comme une œuvre poétique. ${ }^{57}$

Le représentant principal de la théorie du polysystème, Gideon Toury, connu en Occident pour avoir introduit en premier le terme « norme » dans la théorie de la traduction, dit dans un entretien à Toronto en 2003 qu'il a pris connaissance du terme " norme de traduction » de Levý en 1974 par l'intermédiaire de la thèse de doctorat d'Itamar Even Zohar, son maître..$^{58}$ Un autre chercheur à reprendre les idées de Levý est André Lefevere, le fondateur de l'école de manipulation, qui se sépare de la théorie du polysystème dans les années quatre-vingt. En faisant référence à Levý, Lefevere rappelle que le traducteur doit bien connaître la littérature de son pays d'origine pour pouvoir en « combler » des lacunes. ${ }^{59}$ Cette idée correspond aux explications que Levý fournit dans le chapitre «La relation ambiguë envers la littérature d'origine $»$.

Il est évident que d'autres écoles et théoriciens de la traduction ont pris l'œuvre de Levý pour modèle. Ce phénomène concerne par exemple l'école traductologique néerlandaise (the Low-Countries School) et l'école de Nitra en Slovaquie. ${ }^{60}$ Otto Kade, le fondateur de l'École traductologique de Leipzig, fait également référence à L'Art de la traduction, ce qui vaut non seulement pour l'édition allemande, mais aussi pour l'édition tchèque de 1963 . Selon lui, cet ouvrage représente la tentative la plus significative d'élaborer une théorie de la traduction littéraire.

L'Art de la traduction se forme au milieu des années soixante, pratiquement en parallèle avec les travaux théoriques de Georges Mounin, John Catford et Eugene Nida. ${ }^{61}$ Mais pendant longtemps, l'œuvre de Levý est restée à l'écart, et ce pour plusieurs raisons. La première d'entre elles dépend de la situation politique qui, à

\footnotetext{
56 JeTTMAROVÁ in LeVÝ, 2012, p. 8-9.

57 ŠMrHA, 2015, p. 23-24.

58 JETTMAROVÁ, 2016, p. 106

59 ŠMrHA, 2015, p. 40.

60 JetTMAROVÁ, 2016, p. 7.

${ }^{61}$ JetTMarovÁ, 2016, p. 9.
} 
l'époque des deux premières éditions tchèques, limite fortement le contact avec les universitaires au-delà des frontières du bloc de l'Est. L'inaccessibilité des travaux de Levý découle également du fait qu'il les a écrits en tchèque, une langue minoritaire. Une autre raison expliquant la méconnaissance de sa théorie provient d'une mauvaise classification théorico-historique dans le contexte de la traductologie occidentale. Étant novateurs du point de vue théorique, mais surtout méthodologique, les travaux de Levý ne vont pas de pair avec la chronologie des tournants paradigmatiques. La base théorique de L'Art de la traduction vient du structuralisme tchèque dont la traductologie occidentale a faussement interprété les principes, jugeant ce courant comme un prolongement du formalisme russe ou de la théorie du polysystème. De manière plus ou moins implicite, cette perspective classe Levý dans le structuralisme français, à l'époque déjà dépassé. Suivant cette présomption, le structuralisme tchèque est également considéré comme obsolète.

Levý apparaît dans les ouvrages "mainstream» sous l'entrée «théorie des jeux », mais son nom ne figure jamais sous les entrées «norme », « fonction » ou bien «équivalence ». ${ }^{62}$ Dans International Encyclopedia of Translation Studies, l'une des plus grandes encyclopédies traductologiques, il est mentionné 45 fois, la plupart du temps en relation avec la traduction anglaise de son article Překlad jako rozhodovaci proces (Translation as a Decision Process). ${ }^{63}$

\section{Jiří Levý et L'Art de la traduction en milieu francophone}

En ce qui concerne le milieu francophone, l'intérêt pour le travail de Levý s'observe dès le début du XXI ${ }^{\mathrm{e}}$ siècle. Cependant, nous pouvons remarquer de rares mentions avant l'an 2000. La première d'entre elles fait déjà son apparition en 1951 dans la Revue des études slaves et concerne son examen critique des diverses traductions de «Máj» de Mácha. ${ }^{64}$ Son nom figure dans cette revue de façon récurrente. Par exemple, dans l'édition de 1965 il est brièvement mentionné que Levý avait publié les résultats partiels d'une analyse informationnelle du vers. ${ }^{65}$ En 1967, Claude Bremond fait référence au traductologue tchèque dans son article « La conférence de sémiotique de Kazimierz » écrit à la suite de la conférence organisée en 1966 en Pologne. Bremond y évoque l'idée originale qu'a eue Levý d'appliquer la théorie des jeux à la création poétique avant de se pencher quelque peu sur le contexte de son œuvre, notamment pour ce qui est de la versologie et de la sémiotique. $^{66}$

Malgré un certain regain d'intérêt à l'égard de la découverte des idées de Levý, il est actuellement impossible de parler d'une approche systématique. Dans la publication française Introduction à la traductologie : penser la traduction : hier, aujourd'hui, demain (2011) de Mathieu Guidère, le nom de Jiří Levý est mentionné

\footnotetext{
62 JeTTMAROVÁ, 2016, p. 95-99.

${ }^{63}$ Schultze, 2015, p. 109.

${ }^{64}$ Vaillant André, et al. Publications. In: Revue des études slaves, tome 28, fascicule 1-4, 1951, p. 238

${ }^{65}$ Vaillant André, et al. Publications. In: Revue des études slaves, tome 44, fascicule 1-4, 1965 , pp. 286.

${ }^{66}$ Bremond Claude. La Conférence de sémiotique de Kazimierz. In: L’Homme, 1967, tome 7 n² 2 , p. 124 
uniquement en relation avec son article «Translation as a decision process » et la théorie des jeux. ${ }^{67} \mathrm{Il}$ est à noter que la plupart des sources en langue française ont été écrites par des chercheurs et chercheuses tchèques. En 2014, Zuzana Raková a publié son manuel "Les Théories de la traduction », un ouvrage contenant le plus d'informations complètes sur l'activité scientifique de Levý. Elle décrit de manière détaillée L'Art de la traduction ainsi que d'autres écrits fondamentaux de Levý, notamment la publication České teorie překladu [Les Théories tchèques de la traduction] et l'étude Translation as a decision process. Raková y appréhende l'œuvre du traductologue tchèque dans un contexte historico-littéraire plus large. En effet, elle esquisse toute l'évolution de la traductologie tchèque en mettant l'accent sur le structuralisme tchèque. Une autre chercheuse tchèque qui sensibilise le public francophone au travail de Levý est Jovanka Šotolová. En 2016, son article « La littérature française à travers ses traductions tchèques au cours des 25 dernières années » a été publié dans la revue électronique Mutatis Mutandis, faisant partie d'un exemplaire dédié entièrement à l'œuvre de Jiř́i Levý. Šotolová cite également Levý (notamment son ouvrage Les Théories tchèques de la traduction) dans le recueil Histoire de la traduction littéraire en Europe médiane paru en 2019. Plusieurs pages y sont consacrées à sa conception théorique de la traduction. ${ }^{68}$

En 2015, Essam Al-Mohaya soutient sa thèse de doctorat à l'ESIT intitulée « L'Explication en traduction » et dont l'objectif est d'explorer le phénomène de l'explication en traduction. L'auteur y cite la version anglaise de L'Art parue en 2011. Un chapitre entier porte sur la stratégie minimax ; l'explication des relations logiques et la surexplication en traduction en tant que notions introduites par Levý constituent d'autres objets d'étude ${ }^{69}$ Au cours de la dernière décennie, le nom de Levý fait son apparition dans les articles publiés par des personnes natives francophones. Citons à titre d'exemple: «Quand "la traduction est un jeu" : métaphores ludiques dans le discours contemporain sur la traduction » (2013). Encore une fois, l'auteur, Fabio Regattin y mentionne Levý par rapport à la théorie des jeux : il étudie la conception du jeu en traductologie, ses règles et limitations. Il poursuit en énumérant les chercheurs qui ont repris le concept de traduction en tant que jeu à information parfaite introduit par Levý. Certains théoriciens français ne figurent cependant pas dans la liste. L'article « De la traduction aux sciences de la traduction ? », étudiant l'évolution de la pensée traductologique, est paru en 2016 dans le périodique Revue française de linguistique appliquée. Maryvonne Boisseau y signale une méconnaissance des traditions belge, suisse, italienne, allemande, russe, tchèque et slovaque, en mentionnant brièvement L'Art de la traduction et Translation as a decision process.

\section{Les traductions de L'Art de la traduction en d'autres langues}

Même si jusqu'à présent, «l'export anglais » du structuralisme tchèque et de la pensée de Levý est très modeste, ses théories se montrent non seulement à la hauteur des derniers tournants au sein des sciences humaines, mais proposent aussi des

${ }^{67}$ GUIDĖRE, 2011, p. 74

${ }^{68}$ Chalvin, 2019, p. 425.

${ }^{69}$ Al-Mohaya, 2015, p. 76. 
perspectives ouvertes à de nouvelles recherches. ${ }^{70}$ Le regain d'intérêt pour la pensée traductologique de provenance slave, $\mathrm{y}$ compris les théories tchèques, en témoigne. $^{71}$

Ces dix dernières années voient apparaître plusieurs traductions de L'Art de la traduction. En 2011, Filipe Mendes Neckel, qui a largement contribué à répandre les idées de Levý en traductologie latino-américaine, traduit la première partie de cet ouvrage en portugais dans le cadre de sa thèse de doctorat. Sa traduction est publiée en version bilingue, avec le texte allemand en vis-à-vis. Il a décidé de procéder selon la méthode de Schleiermacher, dite « fidèle », et a pris pour l'original la version en langue allemande, d'où le fait qu'il ne substitue pas les exemples cités par des analogies portugaises. En effet, des extraits de littérature allemande restent en version originale, leur traduction en portugais apparaissant dans les notes de bas de page.

En 2013, des chapitres sélectionnés de L'Art ont été traduits en espagnol et publiés dans le recueil Jiř́ Levý: una concepción (re)descubierta comportant plusieurs autres articles et publications traduits par Jana Králová et Miguel Cuenca Drouhard. ${ }^{72}$ Le public hispanophone a toutefois pu prendre connaissance de son œuvre à partir des années quatre-vingt-dix. En effet, Miguel Angel Vega a inclus les thèses de Jiří Levý dans son ouvrage Textos clásicos de la teoría de la traducción (1994). Il avait à sa disposition l'édition allemande de L'Art de la traduction. ${ }^{73}$

L'ouvrage fondamental de Levý sollicite de plus en plus d'attention à l'étranger. C'est en premier lieu grâce à sa traduction anglaise que L'Art de la traduction s'impose en Chine. En 2018, la maison d'édition chinoise China Intercontinental Press publie la version anglaise de l'Art avec une préface en langue chinoise. ${ }^{74}$ Pour ce qui est du continent africain, sa traduction anglaise est utilisée par exemple dans le cadre des cours de traduction à l'Université de Namibie ou en Tanzanie à l'Université de Dar es Salaam. ${ }^{75}$ Concernant l'Amérique latine, la pensée de Levý est connue non seulement au Brésil, mais également en Colombie, où son nom apparaît dans l'anthologie Reseña de libro: Introducción a la traductología: Autores, textos y comentario rédigée par Sergio Bolaños. Il y introduit toute la première partie de L'Art de la traduction, à l'exception des chapitres « La Traduction des pièces de théâtre » et «La Traduction en tant que problème de historico-littéraire $\gg{ }^{76}$

\footnotetext{
70 JeTtMarová, 2016, p. 95.

${ }^{71}$ KrÁlovÁ in MračKOVÁ VAVROUŠOVÁ, 2018, p. 144.

72 MraČKová VAVROUŠOVÁ, 2018, p. 147-149.

${ }^{73}$ MARKSOVÁ, 2015, p. 16-18.

${ }^{74}$ En témoigne par exemple l'article suivant : Cong Lin, Jiri Levy's Conception of Translation: http://qikan.cqvip.com/Qikan/Article/Detail?id=1002270088; ou la traduction anglaise de L'Art de la traduction publiée en Chine : https:/www.amazon.com/Art-Translation-Chinese-XuYuanchong/dp/7508536770.

${ }^{75}$ Communication orale avec les professeurs de traduction : Kristomus Faraja en Tanzanie et Marianne Zappen-Thomson en Namibie, effectuée lors des séjours de recherche dans les deux pays en 2018 et 2019.

${ }^{76}$ BOLAÑOS, 2016, p. VII-XV.
} 


\section{Conclusions}

Dans le présent article, nous avons esquissé l'évolution de L'Art de la traduction de Jiří Levý à partir des années cinquante du siècle dernier jusqu'aux années deux mille dix. La première édition de cet ouvrage (1963) représente à l'époque l'aboutissement de la recherche interdisciplinaire de longue durée, dont les résultats partiels se reflètent dans de nombreux articles, conférences, polycopiés ainsi que des notes manuscrites. L'introduction de L'Art de la traduction à l'étranger, d'abord auprès de lecteurs allemands et russes, marque un tournant décisif dans sa genèse. Étant donné la dépendance de l'original aux littérature et culture tchèques, son adaptation en langue étrangère se caractérise par de nombreuses particularités liées au contenu ainsi qu'aux circonstances accompagnant sa création. La première traduction de L'Art, en langue allemande, joue un rôle clé pour deux raisons : pour la première fois, les thèses et notions de Levý sont dévoilées à la communauté internationale ; elle devient, ensemble avec l'original tchèque, un modèle pour la deuxième édition tchèque rédigée sous la plume de Karel Hausenblas en 1983. Le travail sur cette dernière est remarquable, car elle nécessite une combinaison pertinente des deux versions, la traduction de l'allemand vers le tchèque pour les passages sélectionnés, en même temps qu'un certain nombre de corrections. Elle devient ensuite la base des deux éditions suivantes. Pour des raisons politiques autant que linguistiques, l'œuvre de Levý est pendant longtemps restée quasiment inaccessible à la traductologie mondiale. Néanmoins, nous observons un regain d'intérêt pour celle-ci, survenu au cours de la dernière décennie et dû entre autres aux nouvelles traductions en langue étrangère.

\section{BIBLIOGRAPHIE}

AL-Mohaya Essam (2015), L'explicitation en traduction. Une étude de cas : la traduction du Monde Diplomatique en arabe durant la période 2001-2011. Thèse de doctorat en traductologie. Université Sorbonne Nouvelle - Paris 3. Thèse dirigée par Colette Laplace.

BENEŠOVÁ Rút (2019), Oto Kade a jeho prínos translatologii. Mémoire de master. Univerzita Karlova. Filozofická fakulta. Mémoire dirigé par Tomáš Svoboda.

BOISSEAU Maryvonne (2016), De la traductologie aux sciences de la traduction?, Revue française de linguistique appliquée, vol. XXI, n 1 , pp. 9-21.

BREMOND Claude (1967), La Conférence de sémiotique de Kazimierz, L'Homme, vol. 7, $\mathrm{n}^{\circ}$ 2, pp. 123-125.

Chalvin Antoine, Muller Jean-Léon, TALVISTE Katre, VRINAT-NikOLOV Marie (2019), Histoire de la traduction littéraire en Europe médiane, Rennes, Presses universitaires de Rennes (coll. «Interférences »).

GUIDÈRE Mathieu (2011), Introduction à la traductologie : penser la traduction : hier, aujourd'hui, demain, $2^{\mathrm{e}}$ éd., $2^{\mathrm{e}}$ tirage, Bruxelles, De Boeck.

GRYGAR Mojmír (1999), Terminologický slovnik českého strukturalismu: obecné pojmy estetiky a teorie uměni, Brno, Host.

HAUSENBLAS Karel (1963), Překládání a teorie literatury (Nad knihou J. Levého Umění prèladu), Česká literatura 13, p. 155-168. 
JetTMarovÁ Zuzana (2018), Bibliografie Jiř́ho Levého, AUC Philologica Translatologica Pragensia, $\mathrm{n}^{\circ}$ 2, pp. 185-198.

JetTMAROVÁ Zuzana (2016), Mozaiky překladu, Praha, Karolinum, 2016.

KitzBICHLER Josefine, LUBITZ Katja, MindT Nina (2009), Theorie der Übersetzung antiker Literatur in Deutschland seit 1800, Berlin, Walter de Gruyter.

KITTEL Harald (ed.) (2011), Übersetzung: An international encyclopedia of translation, Berlin, Walter de Gruyter.

LEVÝ Jiř́, ILEK Bohumil (1956), Kapitoly z teorie a metodiky překladu, Praha, Státní pedagogické nakladatelství.

LEVÝ Jiř (1968), Die Übersetzung von Theaterstücken, Babel, vol. 14, nº 2, pp. $77-$ 82.

LEVÝ Jiř́ (1953), O některých zákonitostech překladatelské věrnosti, Slovo a slovesnost, vol. 14, $\mathrm{n}^{\circ}$ 2, pp. 63-80.

LEVÝ Jiř́ (1955), Překladatelský proces - jeho objektivní podmínky a psychologie, Slovo a slovesnost, vol. 16, n 2, pp. 65-87.

LEVÝ Jiř́i (1957), Umělecké otázky překladu, Česká literatura, vol. 5, 1957, nº 4, p. $379-401$.

LEVÝ Jiří (2011) The Art of Translation, traduit par Patrick Corness, Amsterdam/Philadelphia, John Benjamins Publishing Company.

LEVÝ Jiří (1963), Umění překladu, Praha, Československý spisovatel.

LEVÝ Jiř́ (1983), Uměni překladu, $2^{\mathrm{e}}$ édition, Praha, Panorama.

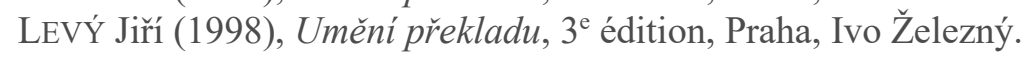

LEVÝ Jiř́ (2012), Uméní překladu, 4 e édition, Praha, Apostrof.

LEVÝ Jiř́ (1958), Úvod do teorie prekladu, Praha, Státní pedagogické nakladatelství.

LeVÝ Jiří (1966), Západni literární věda a estetika, Praha, Československý spisovatel.

LEVÝ Jiř́ (1969), Die literarische Übersetzung: Theorie einer Kunstgattun, traduit par Walter Schamschula. Frankfurt am Main/Bonn, Athenäum Verlag.

LEVÝ Jiří (1974), Iskusstvo perevoda, traduit par Vladimir Rossels, Moskva, Progress.

MARKSOVÁ Romana (2015), Miguel Angel Vega a jeho prínos $k$ rozvoji translatologie. Mémoire de master. Praha, Univerzita Karlova, Filozofická fakulta. Mémoire dirigé par Jana Králová.

MraČKOVÁ VAVROUŠOVÁ Petra (2018), Soudobá recepce díla Jiř́ho Levého ve Španělsku a Iberoamerice, AUC Philologica - Translatologica Pragensia, $\mathrm{n}^{\circ} 2$, pp. 143-153.

POPOVIČ Anton (1983), Originál-preklad: interpretačná terminológia, Bratislava, Tatran

RAKOVÁ Zuzana (2014), Les théories de la traduction, Brno, Masarykova univerzita.

REGATTIN Fabio (2013), Quand « la traduction est un jeu » : métaphores ludiques dans le discours contemporain sur la traduction. TTR, vol. 26, $\mathrm{n}^{\mathrm{o}} 1,1^{\mathrm{er}}$ semestre 2013, pp. 221-254. https://doi.org/10.7202/1036956ar 
SchUltze Brigitte (2015), Jiří Levý's Contribution to Translation Studies as represented in the De Gruyter Encyclopedia Übersetzung, Translation, Traduction, AUC Philologica - Translatologica Pragensia, no 3, pp. 105-112.

ŠMrHA Jan (2015), André Lefevere a jeho manipulační škola. Mémoire. Praha, Univerzita Karlova, Filozofická fakulta. Mémoire dirigé par Zuzana Jettmarová.

ŠoTOLOVÁ Jovanka (2016), La littérature française à travers ses traductions tchèques au cours des 25 dernières années, Mutatis Mutandis: Revista Latinoamericana de Traducción, vol. 9, n 2 (exemplaire dédié à : «The Art of translation » : Jiř́i Levý (1926-1967) y la otra historia de la Traductología), pp. 445-463.

VAiLlant André et al. (1951), Publications, Revue des études slaves, tome 28, fascicule 1-4, pp. 154-296.

VAiLlant André et al. (1965), Publications, Revue des études slaves, tome 44, fascicule 1-4, pp. 163-363. 
- 92 - 